
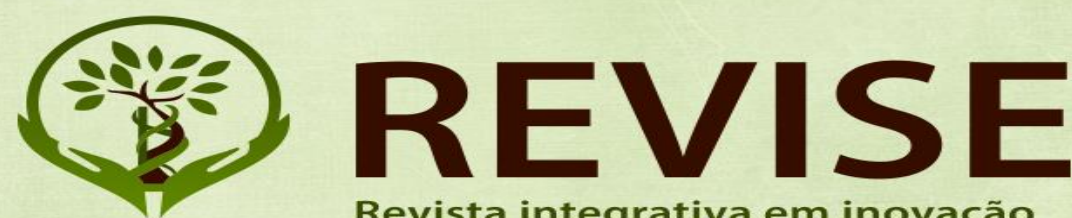

Revista integrativa em inovação tecnológica nas ciências da saúde

ISSN: 2179-6572

\title{
Artigo para discussão: Facilitador do aplicativo e-sus /AB território na unidade de saúde da família Andaiá em Santo Antônio de Jesus-Bahia/Brasil
}

Rafaela Nery Andrade

Tiana Pereira dos Santos Cerqueira

Brenno Lira Sotolani

\begin{abstract}
RESUMO
O artigo registra as apresentações do Congresso internacional de inovação tecnológica nas ciências da saúde: a sustentabilidade das práticas integrativas à agroecologia, mais especificamente a apresentação : Facilitador do aplicativo e-sus /AB território na unidade de saúde da família Andaiá em Santo Antônio de Jesus-Bahia/Brasil. A apresentação versou a respeito de proposta de um aplicativo e- SUS para as equipes do Programa Agente Comunitário de Saúde. O evento aconteceu de 15 a 18 de novembro de 2017 no IFBA SAJ sob a direção da Universidade Federal do Recôncavo da Bahia.

Palavras-chave: Aplicativo. E-SUS. Unidade de Saúde. Facilidades.
\end{abstract}

\begin{abstract}
The article records the presentations of the International Congress of Technological Innovation in Health Sciences: the sustainability of integrative practices in agroecology, more specifically the presentation: e-sus / AB Territory Application Facilitator at the Andaiá Family Health Unit in Santo Antônio de Jesus -Bahia Brazil. The presentation was about the proposal of an e-SUS application for the teams of the Community Health Agent Program. The event took place from November 15 to 18, 2017 at IFBA SAJ under the direction of the Federal University of Recôncavo da Bahia.

Keywords: Application. E-SUS. Health Unit. Facilities.
\end{abstract}



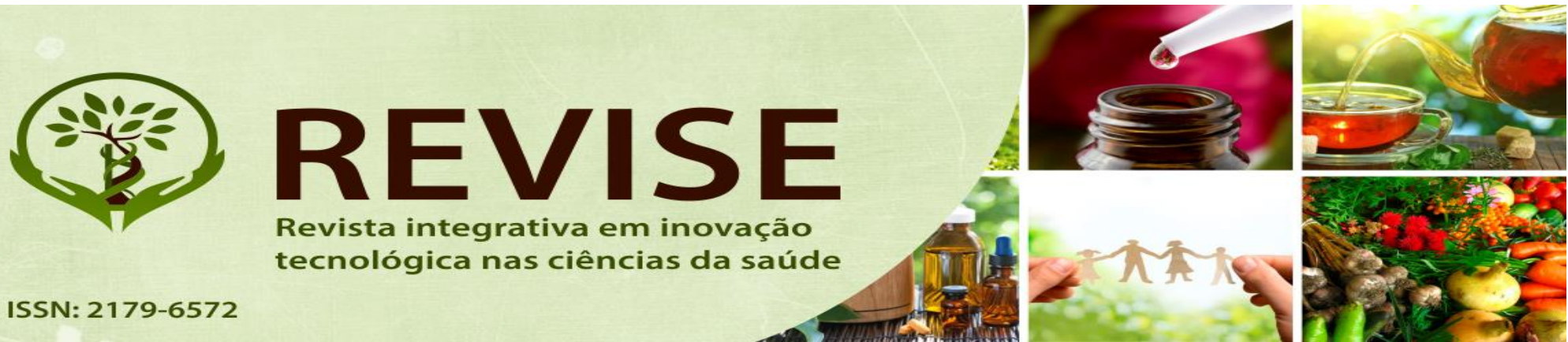

ISSN: 2179-6572

Revista integrativa em inovação tecnológica nas ciências da saúde

\section{INTRODUÇÃO}

O Sistema Único de Saúde (SUS) criado em 1990 pela Lei Orgânica 8.080 é conhecido como um dos maiores sistemas de saúde pública do mundo. Vinculado com a comunidade, cria o Programa de Agentes Comunitários de Saúde (PACS), oficialmente implantado pelo Ministério da Saúde em 1991, tendo como função visitar todas as famílias de uma determinada área da Unidade de Saúde da Família que foi vinculada, serviço organizado e normatizado pela Política Nacional de Atenção Básica abrangendo a promoção, proteção da saúde, prevenção de agravos, diagnóstico, tratamento, reabilitação e redução de danos. Para maior controle e notificação desses agravos relacionados a famílias dispostas em uma comunidade, foram criados métodos sistematizados como o Prontuário Eletrônico do Cidadão (PEC), que objetiva informatizar o fluxo de atendimento realizado pelos profissionais de saúde. Desse modo torna-se necessário reestruturar e integrar as informações da Atenção Básica em nível nacional utilizando meios que reduzam a carga de trabalho na coleta, inserção e uso da informação.

\section{PROBLEMA GERAL}

Portanto esse trabalho tem como objetivo Contribuir na formação e utilização do aplicativo e-SUS AB Território dos Agentes Comunitários de Saúde (ACS) na Unidade de Saúde da Família Andaiá no município de Santo Antônio de Jesus-BA.

\section{PROBLEMA ESPECÍFICO}

Além de realizar formação de noções básicas de informática para os Agentes Comunitários de Saúde da Unidade, serão apresentados o sistema e-SUS AB e o aplicativo e-SUS AB Território e posteriormente os mesmos serão habilitados e treinados no uso do aplicativo. 

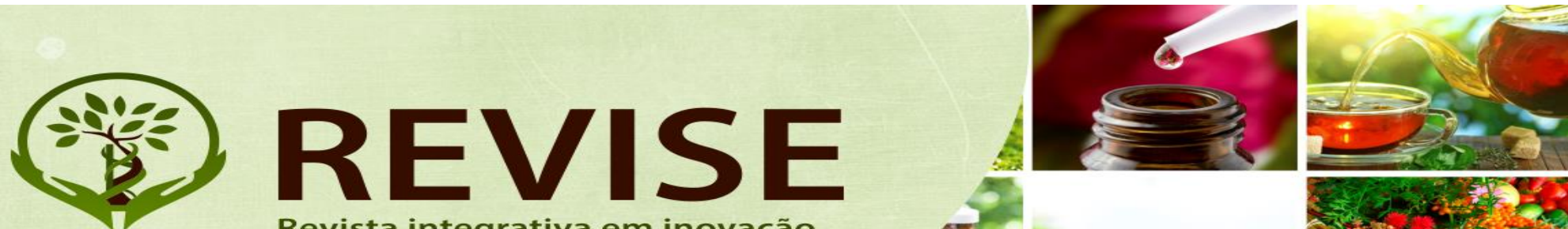

Revista integrativa em inovação tecnológica nas ciências da saúde

ISSN: $2179-6572$

\section{FUNDAMENTAÇÃO TEÓRICA}

No artigo 196 da Constituição Federal Brasileira a saúde é definida como um direito de todos e dever do Estado, juntamente com o Sistema Único de Saúde (SUS) a Constituição respeita seus princípios da universalidade, igualdade e integralidade, logo após a implantação do SUS os serviços de saúde são descentralizados para Estados e Municípios e não somente a nível Federal. Com o passar dos anos o SUS vem avançando e fortalecendo cada vez mais ações e serviços na atenção básica e no desenvolvimento de informatização para monitorar os resultados, a exemplo temos o aplicativo e-SUS AB Território utilizado por meio de tablet proporcionando conforto e segurança aos profissionais, este registra as ações realizadas pelos:

\section{MÉTODO}

Trata-se de um projeto aplicativo que será realizado com os oitos Agentes Comunitários da Unidade de Saúde da Família Andaiá em Santo Antônio de Jesus, para efetivação o mesmo será submetido ao Comitê de Ética e a Secretária Municipal de Saúde. Por meio de um questionário objetivo será identificado o perfil e conhecimentos prévios de informática dos ACS para então realizar o curso básico, após a capacitação os ACS serão treinados in loco no uso do tablet e nas funcionalidades do aplicativo e- SUS AB Território.

\section{CONSIDERAÇÕES FINAIS}

O sistema e-SUS AB Território facilitará o trabalho dos Agentes Comunitários de Saúde e consequentemente resolutividade aos usuários do SUS. Irá agilizar o atendimento ao usuário diminuindo o tempo de espera; qualificando o serviço da Atenção Básica reduzindo gastos ao sistema de saúde a exemplo de impressos. 

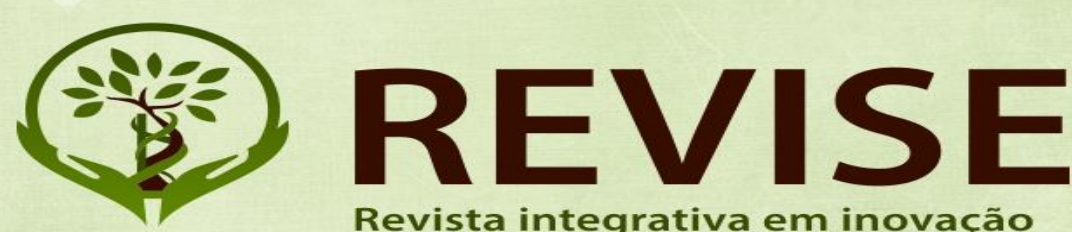

Revista integrativa em inovação tecnológica nas ciências da saúde

\section{REFERÊNCIAS}

BRASIL. Ministério da Saúde. Política Nacional de Atenção Básica. Brasília, 2012. Ministério da Saúde. E-SUS ATENÇÃO BÁSICA. Disponível em: <http://dab.saude.gov.br/portaldab/esus.php> Acesso em: 18 out 2017.

Manual dos Agentes Comunitários de Saúde. O trabalho do agente comunitário de saúde. Brasília, 2009.

Secretaria de Atenção à Saúde- Brasília: Ministério da Saúde, 2015. 